\title{
Cancer of Unknown Primary Presenting With a Giant Shoulder Mass in a Young Man: Prostate Adenocarcinoma
}

\author{
(1) Fatoş Dilan ATílA, ${ }^{1}$ (1) Ömercan TOPALOĞLU, ${ }^{2}$ (i) Emel Ebru PALA, ${ }^{4}$ (i) Çağatay ARSLAN ${ }^{3}$ \\ 'Department of Hematology, Ege University Medical Faculty Hospital, İzmir-Turkey \\ ${ }^{2}$ Department of Endocrinology, İnönü University Medical Faculty Hospital, Malatya-Turkey \\ ${ }^{3}$ Department of Oncology, Medikal Park Hospital, İzmir-Turkey \\ ${ }^{4}$ Department of Pathology, Tepecik Training and Research Hospital, İzmir-Turkey
}

\section{SUMMARY}

Cancer of unknown primary (CUP) is a metastatic tumor for which a standardized diagnostic workup fails to identify the site of origin at the time of making the diagnosis. The most frequent primary origin site is the lung and pancreas (40\%), and the second most frequent primary origin site is the gastrointestinal system. For male patients with CUP, serum prostate specific antigen (PSA) level and free PSA/total PSA ratio should be determined; if necessary, immunohistochemical staining for PSA and P504S should be performed from the metastatic mass. Young patients with CUP should be attentively evaluated to establish the diagnosis and start treatment. We report the case of a young man who presented with a giant shoulder mass of unknown primary site and was diagnosed as having prostate adenocarcinoma.

Keywords: Cancer of unknown primary; metastatic disease; prostate cancer; PSA level.

Copyright $\odot$ 2018, Turkish Society for Radiation Oncology
\end{abstract}

\section{Introduction}

Cancer of unknown primary (CUP) has been traditionally considered as metastatic cancer in the absence of a clinically detectable and anatomically defined primary tumor site after an adequate diagnostic evaluation. CUP accounts for 3-5\% of all malignant epithelial tumors. Metastatic adenocarcinoma is the most common histopathological finding in CUP (80\%). [1] Prostate cancer is considered a disease of older men (aged $>65$ years), but today over $10 \%$ of new diagnoses occur in young men aged $\leq 55$ years. The majority of patients with prostate cancer commonly presented with local symptoms that included urinary complaints, retention, back pain, and hematuria or less frequently with metastatic signs. Here, we report the case of a young man who presented with a giant shoulder mass of unknown primary site and who was diagnosed having prostate adenocarcinoma.

\section{Case Report}

A 49-year old man was admitted with a right shoulder mass and pain that occurred 4 months ago. A physical examination revealed the $10-\mathrm{cm}$ painful mass that restricted right shoulder movement. The patient has had 25 pack-year smoking history. Magnetic resonance imaging was performed for the right shoulder mass. It showed a destructing right scapular mass with soft tissue invasion (Fig. 1). Fine needle aspiration biopsy revealed malignant cytology consistent with metastatic adenocarcinoma (Fig. 2). The PSA level was 300 ng/ml. 18-FDG PET-CT revealed hypermetabolic masses in the prostate gland, paraaortic region, and right shoulder and pathologic activity at the vertebral column. The patient was diagnosed as having metastatic prostate cancer. Radiotherapy was administered to the right shoulder mass and thoracic 7-8 vertebrae. Hormonal treatment with goserelin and bicalutamide were initiated. Addition-

Dr. Fatoş Dilan ATiLLA

Ege Üniversitesi Tıp Fakültesi Hastanesi, Hematoloji,

İmir-Turkey

E-mail: fatosdilankoseoglu@hotmail.com 


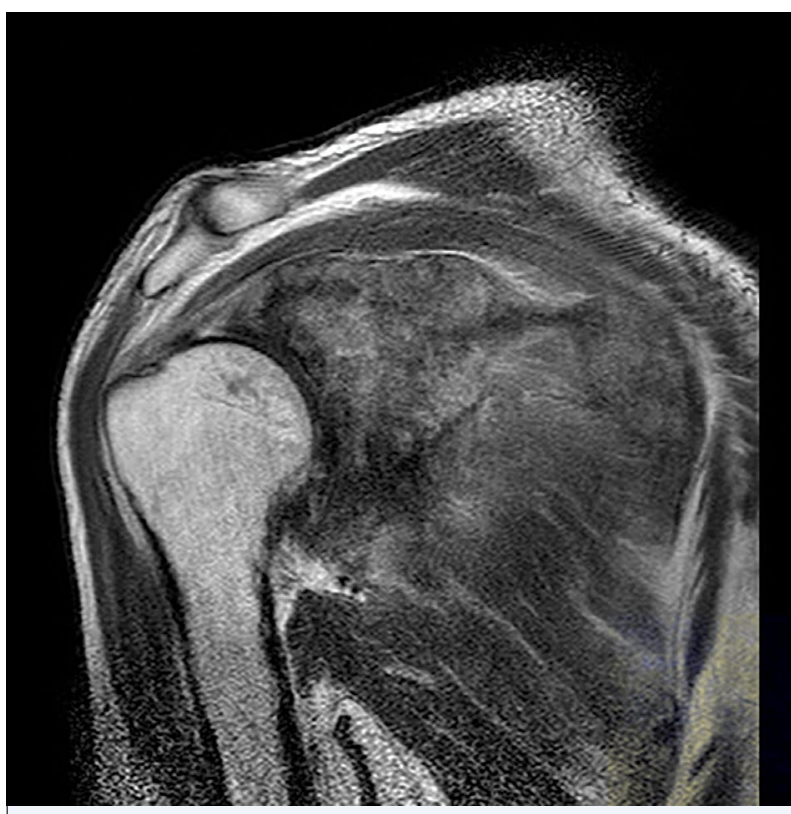

Fig. 1. A coronal T-1 weighted image shows the soft tissue mass on the right shoulder.

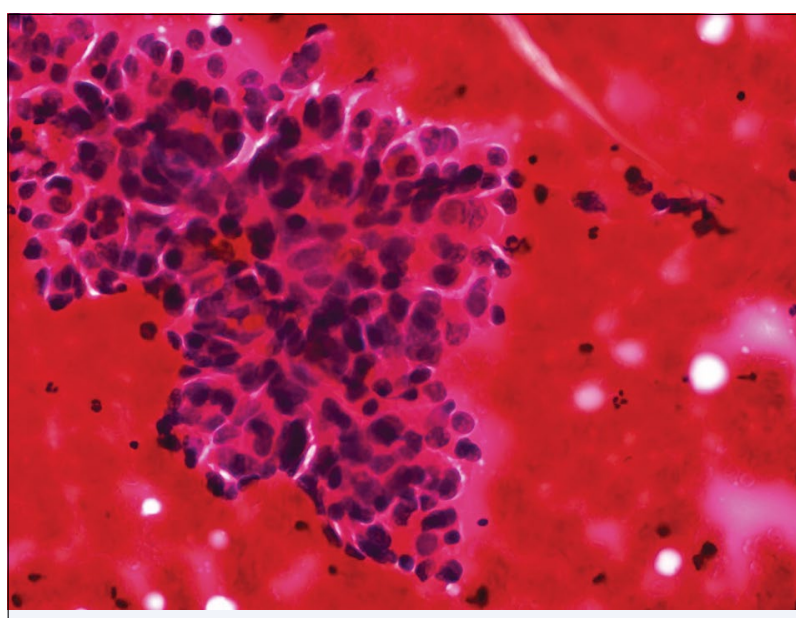

Fig. 2. Fine needle aspiration biopsy shows malignant epithelial cells with hyperchromatic, pleomorphic nuclei and a high nuclear cytoplasmic ratio (HE, 40×).

ally zoledronic acid was initiated to prevent treatmentinduced bone loss. Bicalutamide was stopped after 1 month of treatment. MRI revealed that the shoulder mass shrunk and that the pain regressed after 6 months. The control level of PSA gradually reduced to $0.35 \mathrm{ng} / \mathrm{ml}$ over 6 months. After 10 months of treatment, bone pain increased and bone scintigraphy, chest and abdominal computed tomography scans, and right shoulder MRI revealed disease progression at the bones. However, the PSA level was $1.1 \mathrm{ng} / \mathrm{ml}$ and total testosterone level was
$<20 \mathrm{ng} / \mathrm{dl}$. There was no response to bicalutamide retreatment, and the patient was accepted as metastatic castration resistant prostate cancer. Docetaxel and prednisone were initiated, and goserelin and zoledronic acid were continued. The patient had stable disease at the end of the 10th cycle of docetaxel. He is on follow-up with stable disease after the end of chemotherapy and still receiving goserelin and zoledronic acid. PSA testing and whole body imaging modalities are performing monthly and every 3 months respectively.

\section{Discussion}

CUP is not uncommon. The most frequent primary origin site is the lung and pancreas (40\%), and the second most frequent one is the gastrointestinal system. [2] Metastatic adenocarcinoma is the most common histopathological finding in CUP (80\%).[3] Most patients with unknown primary tumors have poor prognoses with survival often less than 6 months from diagnosis. [4] For male patients with CUP, serum PSA level and free PSA/total PSA ratio should be determined; if necessary, immunohistochemical staining for PSA and P504S should be conducted from the metastatic mass. $[5,6]$ All men older than 40 years with adenocarcinoma or carcinoma not otherwise specified, except those with metastases limited to the liver or brain, should undergo a PSA test, and men presenting with bone metastases or multiple sites of involvement should have their PSA levels assessed regardless of their age.[7] Histopathological comparison of biopsies with specific immunohistochemistry and radiological assessment may act as a guide in the identification of the primary origin site of CUP.[8] The PSA level significantly contributes to the diagnosis of prostate cancer. As in our case, for patients with bone metastases and palpable tumors and with high levels of PSA (>100 ng/ml), prostate gland biopsy is not necessary for making the diagnosis. Metastatic prostate adenocarcinoma is often responsive to hormonal therapy. For patients with metastatic disease, due to a good response $(>80 \%)$, hormonal therapy should be considered. Hormonal therapy is the standard choice in patients with recently diagnosed metastatic prostate cancer. However, the response duration might be lesser in young patients with aggressive histology and the cancer may become metastatic castration resistant with hormonal therapy. From the point of disease control and survival, results of hormonal therapy are similar to those of surgical castration. The response and tolerance to the hormonal therapy should be closely followed in young patients who have metastatic prostate cancer with aggressive histology.[9] 


\section{Conclusion}

In conclusion; unusual presentations of metastatic prostate carcinoma should not mislead clinicians. Metastatic prostate adenocarcinoma might be with poor prognosis in younger patients.

Peer-review: Externally peer-reviewed.

Conflict of Interest: None declared.

Authorship contributions: Concept - F.D.A.; Design F.D.A.; Supervision - Ç.A.; Data collection \&/or processing - F.D.A., Ö.T.; Analysis and/or interpretation - F.D.A., Ö.T., E.E.P.; Literature search - F.D.A.; Writing - F.D.K., Ö.T.; Critical review - Ç.A.

\section{References}

1. Pavlidis N, Pentheroudakis G. Cancer of unknown primary site. Lancet 2012;379(9824):1428-35.

2. Pavlidis N, Briasoulis E, Hainsworth J, Greco FA. Diagnostic and therapeutic management of cancer of an unknown primary. Eur J Cancer 2003;39(14):19902005.

3. Hemminki K, Bevier M, Hemminki A, Sundquist J. Survival in cancer of unknown primary site: popula- tion-based analysis by site and histology. Ann Oncol 2012;23(7):1854-63.

4. Hainsworth JD, Greco FA. Management of patients with cancer of unknown primary site. Oncology (Williston Park) 2000;14(4):563-74.

5. Cho JY, Shim EJ, Kim IS, Nam EM, Choi MY, Lee KE, et al. Cancer of unknown primary finally revealed to be a metastatic prostate cancer: a case report. Cancer Res Treat 2009;41(1):45-9.

6. Sheridan T, Herawi M, Epstein JI, Illei PB. The role of P501S and PSA in the diagnosis of metastatic adenocarcinoma of the prostate. Am J Surg Pathol 2007;31(9):1351-5.

7. National Comprehensive Cancer Netwoek. NCCN Clinical Practice Guidelines in Oncology. Occult Primary (Cancer of Unknown Primary [CUP]). Version 1.2013. Available at: https://www2.tri-kobe.org/nccn/ guideline/archive/occult2013/english/occult.pdf. Accessed Sep 20, 2018.

8. Blaszyk H, Hartmann A, Bjornsson J. Cancer of unknown primary: clinicopathologic correlations. APMIS 2003;111(12):1089-94.

9. Bastide C, Bruyère F, Karsenty G, Guy L, Rozet F. Hormonal treatment in prostate cancer. [Article in French]. Prog Urol 2013;23(15):1246-57. 